myositis. From the clinical point of view, the main pattern of disease presentation was an isolated ILD in all groups at the onset and only in anti-PL12 positive ASSD at last follow-up.
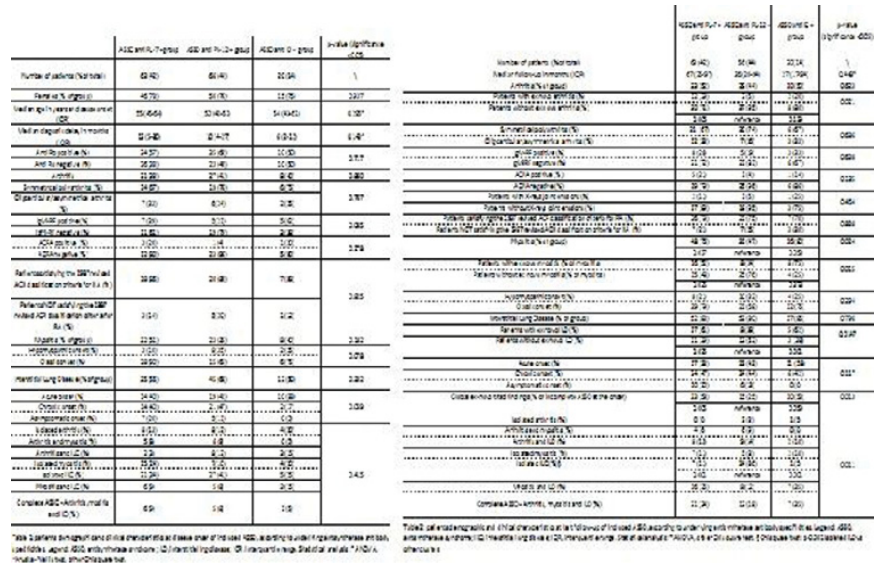

Conclusions: Our study seems to indicate that clinical spectrum time course of anti PL-12 positive ASSD is different from that of anti PL7 and of anti EJ positive ASSD. The clinical pattern associated with these two latter antibodies was very similar. Furthermore, anti PL-12 positive patients seems to have a more stable disease, with a less common occurrence of ex-novo triad findings during the follow-up

References:

[1] Cavagna L. Medicine 2015.

[2] Cavagna L. CRAI 2016.

[3] Cavagna L. ARD 2016 (Abstract).

[4] Trallero Araguas E. Scand J Rheumatol 2016

Acknowledgements: To all members of the AENEAS collaborative group.

Disclosure of Interest: None declared

DOI: 10.1136/annrheumdis-2017-eular.2592

\section{AB0631 THE CLINICAL CONSEQUENCES PRESENCE OF ANTI-PM/SCL ANTIBODIES IN SYSTEMIC SCLEROSIS}

E. Wielosz, M. Dryglewska, M. Majdan. Department of Rheumatology and Connective Tissue Diseases, Medical University of Lublin, Lublin, Poland

Background: Anti-PM/Scl (a-PM/Scl) antibodies are found in different systemic autoimmune disease such as polymyositis, dermatomyositis, systemic sclerosis (SSc), and overlap syndromes. According to literature they are detected in about $2 \%$ of patients with SSc, but their presence are more common in SSc with myositis overlap. Features positively associated with the presence of a-PM/Scl antibodies included younger age at disease onset, skeletal muscle involvement, calcinosis, inflammatory arthritis, and overlap disease. On the other hand interstitial lung disease and gastrointestinal symptoms were less frequent in SSc patients with a-PM/Scl.

Objectives: The aim of the study was to assess the clinical consequences presence of a-PM/Scl antibodies in patients with SSc.

Methods: The study was performed in 126 European Caucasian SSc patients (98-female and 28-male) hospitalized consecutively in the Department of Rheumatology and Connective Tissue Diseases. Patients fulfilled the ACR classification criteria of SSc (59 have diffuse cutaneous SSc and 67 limited SSc). The study group were studied according to the presence of a-PM/Scl antibodies applying commercial test - EUROLINE Systemic Sclerosis Profile. Detection and interpretation of results was carried out electronically using the specific program Euroimmun- EUROLINEScan. The subtype of SSc, incidence of internal organ involvement and serological profile were determined in the whole group. Due to the presence of a-PM/Scl antibodies, patients were divided into two groups a-PM/Scl (+) SSc - 22 pts and a-PM/Scl (-) SSc- 104 pts.

Results: a-PM/Scl antibodies were detected in $22 / 126$ patients with SSc $(17,5 . \%)$ We showed a significant positive association with a-PM/Scl antibodies and myalgia or myosistis $(p=0,0379)$, contractures $(p=0,0002)$ and prevalence of overlap syndrome $(p=0,0142)$. There were no relationship between the presence of a-PM/Scl antibodies and subtypes of SSc, other organ involvement, digital ulcers or calcinosis.

Conclusions: In SSc anti-PM/Scl antibodies are frequently associated with myalgia or myositis, contractures and overlap syndrome.

Disclosure of Interest: None declared

DOI: 10.1136/annrheumdis-2017-eular.4393

\section{AB0632 ASSESSMENT OF VEGF AND OTHER CYTOKINES IN THE TEAR OF PATIENTS WITH SYSTEMIC SCLEROSIS}

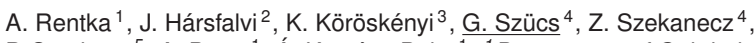
P. Szodoray ${ }^{5}$, A. Berta ${ }^{1}$, Á. Kemény-Beke ${ }^{1}$. ${ }^{1}$ Department of Ophthalmology, University of Debrecen, Faculty of Medicine, Debrecen; ${ }^{2}$ Department of Biopsysics and Radiation Biology, Semmelweis University, Budapest; ${ }^{3}$ Department of Biochemistry and Molecular Biology; ${ }^{4}$ Department of Rheumatology, University of Debrecen, Faculty of Medicine, Debrecen, Hungary; 5 Institute of Immunology, Rikshospitalet, Oslo, Norway

Background: Systemic sclerosis (SSc) is an autoimmune disease, characterized by widespread small vessel vasculopathy, immune dysregulation with production of autoantibodies, and progressive fibrosis. SSc may be associated with sicca syndrome. Changes in levels of proangiogenic and proinflammatory cytokines had already been determined largely in serum, however, the local inflammatory and cytokine milieu in the tear of SSc patients has not yet been evaluated.

Objectives: We wished to determine VEGF and other cytokine and chemokine levels in tear samples of SSc patients.

Methods: First, forty-three patients (40 female and 3 men, mean (SD) age 61 (48-74) years) with SSc and 27 healthy controls were enrolled in the VEGF study. Basal tear sample collection and tear velocity investigations were carried out followed by an ophthalmological examination. Total protein concentrations and VEGF levels were determined in tear samples. In the multiple cytokine study, unstimulated tear samples were collected from nine patients with SSc and 12 age- and gender-matched healthy controls. The relative levels of 102 different cytokines were determined by a cytokine array, and then absolute levels of four key cytokines were determined by a magnetic bead assay.

Results: In the first study, the mean collected tear fluid volume developed 10.4 $L(1.6-31.2)$ in patients and $15.63 \mathrm{~L}(3.68-34.5)$ in control subjects. The mean total protein level was $6.9 \mathrm{~g} / \mathrm{L}(1.8-12.3)$ and $4.1 \mathrm{~g} / \mathrm{L}(0.1-14.1)$ in tear samples of SSc patients and controls, respectively. In patients with SSc, the mean VEGF tear concentration was $4.9 \mathrm{pg} / \mathrm{L}(3.5-8.1)$ compared to $6.15 \mathrm{pg} / \mathrm{L}(3.84-12.3)$ in healthy samples. Multicytokine-array studies revealed shifted cytokine profile characterized by predominance of proinflammatory mediators in the tear samples of SSc patients. Out of the 102 analyzed proteins, nine were significantly increased in tears of patients with SSc. Based on the multiplex bead results, CRP, interferon-inducible protein 10 (IP-10) and monocyte chemoattractant protein-1 $(\mathrm{mcp}-1)$ levels were significantly higher in tears of patients with SSc compared to controls.

Conclusions: Impaired angiogenesis has been found by other investigators in SSc. This is reflected by lower VEGF levels in the tear samples of SSc patients compared to controls. The multi-cytokine array study revealed increased production of CRP and two important pro-inflammatory chemokines in the tear of SSc patients. Our current data depict a group of inflammatory mediators, which may play a significant role in ocular pathology of SSc.

Disclosure of Interest: None declared

DOI: 10.1136/annrheumdis-2017-eular.3218

\section{AB0633 RITUXIMAB IN SCLERODERMA RELATED INTERSTITIAL LUNG DISEASE: A SINGLE CENTRE EXPERIENCE FROM NORTH INDIA}

G. Naidu, S.K. Sharma, V. Dhir, S. Jain. Internal Medicine, PGIMER,

\section{Chandigarh, India}

Background: Pulmonary involvement is one of the major causes of morbidity and mortality in patients with progressive systemic sclerosis (PSS). Clinically significant interstitial lung disease (ILD) is noted in $25 \%$ of patients and accounts for $33 \%$ of deaths in PSS patients $[1,2]$. Cyclophosphamide (CYC) and mycophenolate mofetil (MMF) have been shown to retard progression of ILD [3, 4]. The limited data on rituximab indicate that rituximab might be effective in PSS related ILD [5].

Objectives: To study the efficacy of rituximab in patients with PSS related ILD.

Methods: The clinical details of all patients of PSS related ILD who were treated with rituximab were noted retrospectively from the case files. Forced vital capacity (FVC) value before and 1 year after administration of rituximab were noted. Increase in FVC by $10 \%$ from baseline was considered as improvement and fall in FVC by $10 \%$ or absolute value less than $40 \%$ of predicted was considered as worsening. Patients with FVC $\pm 10 \%$ from baseline were considered to have stabilized lung functions.

Results: A total of 11 patients received rituximab between 2013 and 2016 Six $(54.5 \%)$ patients were females. Median age of the subjects was 44 years (range: $31-75$ years). All patients received intravenous CYC at least 1 year before rituximab administration and had either not responded to CYC or worsened after initial response to CYC. All patients received 2 doses of rituximab (1gram each) at 2 weeks interval. Median FVC before rituximab was $57 \%$ of predicted. Two patients did not have post rituximab FVC values. Median FVC 1 year after rituximab was $54 \%$ predicted. Out of the remaining 9 patients, $2(22.2 \%)$ patients had improvement in FVC, 6 (66.7\%) patients had stabilization of FVC and 1 patient worsened. One patient, who had stabilization of FVC with rituximab expired after 2 years of receiving rituximab.

Conclusions: Rituximab was effective in stabilization of lung functions in patients of PSS with ILD who did not have favourable outcome with intravenous CYC. 
Efficacy of rituximab in treatment naive PSS related ILD patients needs to be studied

\section{References:}

[1] Tyndal AJ, Banert B, Vonk M, Airo P, Cozzi F, Carreira PE et al. Causes and risk factors for death in systemic sclerosis: a study from the EULAR Scleroderma Trials and Research (EUSTAR) database. Ann Rheum Dis. 2010; 69: 1809-152.

[2] McNearney TA, Reveille JD, Fischbach M, Friedman AW, Lisse JR, Goel N et al. Pulmonary involvement in systemic sclerosis: associations with genetic, serologic, sociodemographic and behavioral factors. Arthritis Rheum. 2007; 57: 318-26.

[3] Tashkin DP, Elashoff R, Clements PJ,Goldin J, Roth MD, Furst DEet al. Cyclophosphamide versus placebo in scleroderma lung disease. $\mathrm{N}$ Engl J Med. 2006;354: 2655-66.

[4] Tashkin DP, Roth MD, Clements PJ, Furst DE, Khanna D, Kleerup EC et al. Mycophenolate mofetil versus oral cyclophosphamide in scleroderma-related interstitial ling disease (SLS II): a randomised controlled, double blind, paralle group trial. Lancet Respir Med. 2016; 4: 708-19.

[5] Daoussis D, Melissaropoulos K, Sakellaropoulos G, Antonopoulos I, Markatseli TE, Simopoulou T et al. A multicenter, open-label, comparative study of B-cell depletion therapy with Rituximab for systemic sclerosis-associated interstitial lung disease. Semin Arthritis Rheum. 2016; pii: S0049-0172(16)30344-4.

Disclosure of Interest: None declared

DOI: 10.1136/annrheumdis-2017-eular.5384

\section{AB0634 RITUXIMAB IN SYSTEMIC SCLEROSIS-INTERSTITIAL LUNG DISEASE, A CASE SERIES OF 18 PATIENTS}

G. Guzelant ${ }^{1}$, M. Melikoglu ${ }^{1}$, B. Musellim ${ }^{2}$, D.D. Yilmaz ${ }^{2}$, I. Fresko ${ }^{1}$, E. Seyahi ${ }^{1}$, G. Hatemi ${ }^{1}$, S. Ugurlu ${ }^{1}$, V. Hamuryudan ${ }^{1} .{ }^{1}$ Cerrahpasa Medical Faculty Department of Internal Medicine Division of Rheumatology; ${ }^{2}$ Cerrahpasa Medical Faculty Department of Pulmonology, Istanbul University, Istanbul, Turkey

Background: Interstitial lung disease (ILD) is a severe complication of systemic sclerosis (SSc). Immunosuppressives such as cyclophosphamide (CYC) and mycophenolate mophetil (MMF) are used in its treatment with no proven efficacy (1). Rituximab (RTX) appears to be an emerging agent according to case series. Objectives: This retrospective study aims to evaluate the efficacy of RTX on SSc-ILD in a group of patients followed in our center.

Methods: A chart review revealed 18 patients (16 women, 2 men; mean age $50.3 \pm 12.1$ SD years (range $30-72$ ), mean disease duration $8.3 \pm 9.3$ SD years) with SSc who have been diagnosed as having ILD (confirmed by high-resolution thorax computed tomography and pulmonary function tests) and have been treated with one or more cycles of RTX. Efficacy was evaluated according to the criteria of the American Thoracic Society: improvement= an increase in $\mathrm{FVC} \geq 10 \%$ or DLCO $\geq 15 \%$; worsening= a decrease in $\mathrm{FVC} \geq 10 \%$ or $\mathrm{DLCO} \geq 15 \%$; stabilization= changes in FVC less than $10 \%$ or DLCO less then $15 \%$ (2).

Results:

Table 1 Demographic findings of the patients and their response to RTX treatment

\begin{tabular}{lccc}
\hline & $\begin{array}{c}\text { Group 1 } \\
\text { (ILD with short duration } \\
\text { and naive to treatment) }\end{array}$ & $\begin{array}{c}\text { Group 2 } \\
\text { (ILD with long duration } \\
\text { and previous IS therapy) }\end{array}$ & All patients \\
\hline Number of patients & $4(22.2 \%)$ & $14(77.7 \%)$ & 18 \\
Sex (F/M) & $3 / 1$ & $13 / 1$ & $16 / 2$ \\
Mean disease duration & $2 \pm 0.8$ SD years & $10.2 \pm 9.8$ SD years & $8.3 \pm 9.3$ SD years \\
Follow-up time after & & & \\
$\quad$ initiation of RTX & $12.2 \pm 6.8$ SD months & $21 \pm 12.4$ SD months & $19 \pm 11.8$ SD months \\
Baseline FVC\% & $69.2 \pm 20.9$ & $65.1 \pm 14$ & \\
Last FVC\% & $66.7 \pm 13$ & $61.6 \pm 19.6$ & \\
Baseline DLCO\% & $57.7 \pm 24.1$ & $43.5 \pm 12.2$ & \\
Last DLCO\% & $52 \pm 18$ & $41.2 \pm 21.8$ & \\
Outcome: Stable/ & & & \\
$\quad$ Improvement $(\mathrm{n})$ & 2 & 7 & 7 \\
Worsening (n) & 2 & 5 & 1 \\
Death & 0 & 1 & 1 \\
Unable to do PFT (n) & 0 & 1 & \\
\hline
\end{tabular}

Four patients were treatment naive for ILD when they received RTX (Group 1). The mean duration between the diagnosis of ILD and RTX treatment in Group 1 was 3.5 months (range 0-14 months). The average RTX cycle in this group was 2 with 1 patient also receiving mycophenolate mophetil in combination with RTX. The mean follow-up time after the initiation of RTX in this group was $12.2 \pm 6.8$ SD months (range 7-22 months). FVC/DLCO was stable or improved in 2/4 compared to baseline and worsened in 2/4 at the end of follow-up at group 1.

Fourteen patients had a 10.2 years-history of SSc and have been treated with immunosuppressives (cyclophosphamide, azathioprine, methotrexate, MMF) for ILD before RTX (Group 2). The mean duration between the diagnosis of ILD and RTX treatment in Group 2 was 71.2 months (range 5-246 months). These patients received a mean of 3 cycles of $\mathrm{RTX}$ with 5 receiving MMF $(n=3)$ or AZA $(n=2)$ in addition to RTX. One patient died after 3 months following the first RTX cycle (unknown reason) and 1 was unsuitable for spirometry because of microstomia. Of the remaining 12 patients in Group 2, improvement or stabilisation was seen in 7 and worsening was seen in the remaining 5 patients.

Conclusions: RTX appears to be modestly effective for ILD of SSc. The duration of ILD as well as the presence or absence of previous immunosuppressive therapy do not appear as playing a role in response.

References:

[1] Avouac J et al. EULAR Scleroderma Trials and Research group (EUSTAR) recommendations for the treatment of systemic sclerosis. Ann Rheum Dis 2009.

[2] An Official ATS/ERS/JRS/ALAT Statement: IPF: Evidence-based Guidelines for Diagnosis and Management.

Disclosure of Interest: None declared

DOI: 10.1136/annrheumdis-2017-eular.4908

\section{AB0635 THE CLINICAL FEATURES AND PROGNOSIS OF PULMONARY ARTERIAL HYPERTENSION ASSOCIATED WITH SCLERODERMA AND OTHER CONNECTIVE TISSUE DISEASE DURING THE MODERN MEDICAL ERA IN JAPAN}

H. Maki ${ }^{1}$, M. Hatano ${ }^{2}$, S. Minatsuki ${ }^{1}$, T. Inaba ${ }^{1}$, I. Komuro ${ }^{1}$, Y. Asano ${ }^{3}$, S. Sato ${ }^{3} .{ }^{1}$ Cardiovascular Medicine; ${ }^{2}$ Therapeutic Strategy for Heart Failure; ${ }^{3}$ Dermatology, The University of Tokyo, Tokyo, Japan

Background: Due to the recent progress of vasodilators for pulmonary arterial hypertension (PAH), the prognosis of PAH has been getting better for the past 10 years. Among them, idiopathic PAH (IPAH) well improved but PAH associated with connective tissue disease (CTD-PAH) especially scleroderma (SSc-PAH) have poorer prognosis than IPAH.

Objectives: This study intended to clarify the clinical features and prognosis of CTD-PAH in modern era when multiple PAH drugs are available, in addition, compare between SSc-PAH and PAH associated with other CTD-PAH (non SSc-PAH).

Methods: Fifty-seven consecutive CTD-PAH patients were enrolled to this study, who received hemodynamic examination with right heart catheterization between 2004 and 2016. Thirty of 57 patients were SSc-PAH patients and other 27 patients were non SSc-PAH patients ( 11 mixed connective tissue disease, 8 systemic lupus erythematosus, 5 primary sjogren syndrome, 1 polymyositis, 1 rheumatoid arthritis and 1 Still's disease were included). We retrospectively analyzed the relationship between clinical parameters at baseline and the prognosis of CTD-PAH patients. Results: Mean age at entry were $65.3 \pm 10.3$ and $48.3 \pm 15.5$ years old each other (SSc-PAH vs non SSc-PAH P<0.001). Twenty-eight SSc-PAH (93\%) and 26 non SSc-PAH patients (96\%) took at least one vasodilator, among them, $18 \mathrm{SSc}-\mathrm{PAH}$ $(60 \%)$ and 13 non-SSc-PAH (48\%) patients took multiple vasodilators at the end of follow-up period. Comparing the baseline clinical parameters between two groups, vital capacity and diffusing capacity of the lung for carbon monoxide (DLCO) were significantly lower in SSc-PAH patients than non SSc-PAH patients and brain natriuretic peptide and creatinine level were higher in SSc-PAH patients than non SSc-PAH patients $(\mathrm{P}<0.05)$. However, there were no significant differences in hemodynamic indices between two groups. During a mean follow-up period of $42.5 \pm 31.5$ months, 22 patients (18 SSc-PAH and 4 non SSc-PAH) died or received lung transplantation. The SSc-PAH patients had worse prognosis than non SSc-PAH patients (figure: $\mathrm{P}<0.001$ ). Only 6 of 18 SSc-PAH patients and 1 of 4 non SSc-PAH patient died of $\mathrm{PH}$ related cardiovascular event and other principal causes of death included interstitial lung disease (ILD), neoplasm and infection. Applying multivariate Cox-proportional hazard regression, mean pulmonary arterial pressure, right atrial pressure, creatinine level and \%DLCO were extracted as the independent risk for all-cause mortality.

(survival rate)

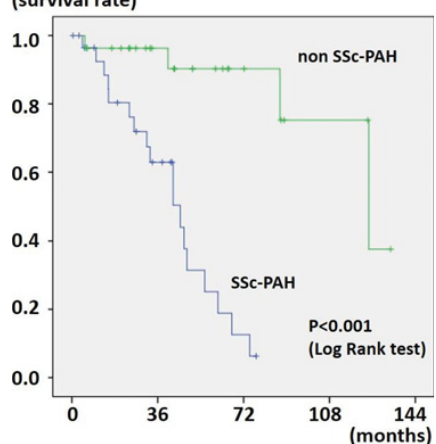

Conclusions: SSc-PAH patients had poor prognosis among CTD-PAH patients despite the progression of PAH drugs. As well as the report from REVEAL registry, not only PAH severity but also respiratory dysfunction may predict the prognosis of CTD-PAH patient. Scleroderma is multiorgan disease affected by complex pathology of vasoconstriction (ischemia), proliferation, inflammation, autoimmune disorder and fibrosis. Our data suggest that vasodilator alone is not enough to improve the prognosis of CTD-PAH patients. Comprehensive therapeutic strategy for scleroderma is needed.

\section{References:}

[1] Chung L.et al. Unique predictors of mortality in patients with pulmonary arterial hypertension associated with systemic sclerosis in the REVEAL registry. Chest 2014;146(6):1494-1504. 